\title{
Grape seed extract supplementation prevents high-fructose diet-induced insulin resistance in rats by improving insulin and adiponectin signalling pathways
}

\author{
Aramsri Meeprom ${ }^{1}$, Weerachat Sompong ${ }^{1}$, Wannaporn Suwannaphet ${ }^{2}$, Sirintorn Yibchok-anun ${ }^{2}$ \\ and Sirichai Adisakwattana ${ }^{3,4 *}$ \\ ${ }^{1}$ Program in Clinical Biochemistry and Molecular Medicine, Department of Clinical Chemistry, Faculty of Allied Health \\ Sciences, Chulalongkorn University, Bangkok, Thailand \\ ${ }^{2}$ Department of Pharmacology, Faculty of Veterinary Science, Chulalongkorn University, Bangkok, Thailand \\ ${ }^{3}$ Program in Nutrition and Dietetics, Faculty of Allied Health Sciences, Chulalongkorn University, Bangkok, Thailand \\ ${ }^{4}$ Department of Transfusion Medicine, Faculty of Allied Health Sciences, The Medical Food Research and Development \\ Center, Chulalongkorn University, Bangkok, Thailand
}

(Received 1 October 2010 - Revised 28 February 2011 - Accepted 4 March 2011 - First published online 31 May 2011)

\begin{abstract}
Recent evidence strongly supports the contention that grape seed extract (GSE) improves hyperglycaemia and hyperinsulinaemia in highfructose-fed rats. To explore the underlying molecular mechanisms of action, we examined the effects of GSE on the expression of muscle proteins related to the insulin signalling pathway and of mRNA for genes involved in the adiponectin signalling pathway. Compared with rats fed on a normal diet, high-fructose-fed rats developed pathological changes, including insulin resistance, hyperinsulinaemia, hypertriacylglycerolaemia, a low level of plasma adiponectin and a high level of plasma fructosamine. These disorders were effectively attenuated in high-fructose-fed rats supplemented with GSE. A high-fructose diet causes insulin resistance by significantly reducing the protein expression of insulin receptor, insulin receptor substrate-1, Akt and GLUT4, and the mRNA expression of adiponectin, adiponectin receptor R1 (AdipoR1) and AMP-activated protein kinase (AMPK)- $\alpha$ in the skeletal muscle. Supplementation of GSE enhanced the expression of insulin signalling pathway-related proteins, including Akt and GLUT4. GSE also increased the mRNA expression of adiponectin, AdipoR1 and AMPK- $\alpha$. In addition, GSE increased the mRNA levels of glycogen synthase and suppressed the mRNA expression of glycogen synthase kinase-3- $\alpha$, causing an increase in glycogen accumulation in the skeletal muscle. These results suggest that GSE ameliorates the defective insulin and adiponectin signalling pathways in the skeletal muscle, resulting in improved insulin resistance in fructose-fed rats.
\end{abstract}

Key words: Grape seed extract: Fructose: Insulin signalling: Adiponectin signalling: Insulin resistance

Insulin resistance is a growing and significant health problem strongly associated with obesity, dyslipidaemia and type 2 diabetes mellitus. An increase in the consumption of high-sugar diets, especially of fructose, correlates positively with the development of hyperinsulinaemia, insulin resistance, hypertension and hypertriacylglycerolaemia ${ }^{(1)}$. Emerging evidence clearly indicates that chronic feeding of a fructose-enriched diet markedly alters insulin signal transduction involving receptor autophosphorylation and tyrosine kinase activity, and results in post-receptor defects at the level of phosphorylation substrates or effector molecules such as GLUT4, leading to increased blood circulation of glucose ${ }^{(2,3)}$. Recently, it has been reported that long-term high-fructose feeding is associated with a decrease in plasma adiponectin levels and defects in adiponectin receptor and AMP-activated protein kinase (AMPK) expression in skeletal muscles and adipocytes, leading to impaired GLUT4 translocation to the plasma membrane ${ }^{(4,5)}$. In addition, impairment in adiponectin and AMPK signalling has also been shown to contribute to alterations in glucose metabolism and impairment of insulin sensitivity ${ }^{(6)}$.

Recently, it has been found that the consumption of plant foods has been associated with a reduced risk of developing chronic diseases such as diabetes mellitus and CVD. In the prevention or treatment of a variety of diseases, grape seed extract (GSE) has been shown to have various beneficial pharmacological effects, including anti-hyperlipidaemic ${ }^{(7)}$,

\footnotetext{
Abbreviations: AdipoR1, adiponectin receptor R1; AMPK, AMP-activated protein kinase; AST, aspartate transaminase; GSE, grape seed extract; GS1, glycogen synthase-1; GSK-3, glycogen synthase kinase-3; HF, high-fructose diet; Hprt, hypoxanthine guanine phosphoribosyl transferase; IR, insulin receptor; IRS-1, insulin receptor substrate-1; ND, normal diet.
} 
anti-inflammatory $^{(8)}$ and antibacterial activities ${ }^{(9)}$. GSE and its bioactive compounds exhibit favourable anti-diabetic effects by stimulating glucose uptake in insulin-sensitive cell lines ${ }^{(10)}$, inhibiting intestinal $\alpha$-glucosidases, pancreatic $\alpha$-amylase activities and the process of glycation ${ }^{(11)}$. A recent study has clearly shown that oligomeric procyanidins in GSE stimulate glucose uptake related to Akt, p44/42 and p38 mitogen-activated protein kinase function, which differs from insulin activation and results in differences in downstream signalling ${ }^{(12)}$. Our previous study demonstrated that GSE improved glucose tolerance and prevented insulin resistance in high-fructose diet (HF)-induced diabetic rats. In addition, GSE significantly increased the activities of hepatic superoxide dismutase and catalase and significantly suppressed lipid peroxidation in HF-fed rats ${ }^{(13)}$. On the basis of these findings, it can be speculated that GSE may improve insulin sensitivity by regulating the insulin signalling or adiponectin signalling pathway in HF-induced diabetic rats. To reveal the underlying mechanisms at the molecular level, the present study was undertaken to investigate the protein expression of the insulin signalling pathway and the mRNA expression of the adiponectin signalling pathway in highfructose-fed rats.

\section{Materials and methods}

\section{Chemicals}

$o$-Dianisidine dihydrochloride and peroxidase-glucose oxidase (PGO) enzymes were purchased from Sigma-Aldrich Company (St Louis, MO, USA). Cholesterol, TAG and HDLcholesterol kits were obtained from Human Diagnostics (Wiesbaden, Germany). Anti-insulin receptor- $\beta$ (IR $\beta$ ), antiinsulin receptor substrate-1 (IRS-1), anti-Akt, anti-GLUT4 and anti-IgG horseradish peroxidase-linked antibodies were purchased from Cell Signaling Technology (Beverly, MA, USA). TRIzol reagent and DNase1 were obtained from Invitrogen (Carlsbad, CA, USA) and Promega (Madison, WI, USA), respectively. All PCR primers and oligo dT primers were obtained from Pacific Science (Anaheim, CA, USA). The Rat Adiponectin ELISA kit was purchased from Adipogen (Incheon, Korea). All other chemical reagents used in the present study were of analytical grade.

\section{Preparation of grape seed extract}

The extraction and phytochemical analysis of grape seeds were performed according to a previously established method $^{(14)}$. The chemical composition of GSE included $50 \cdot 1 \%$ total flavanols, $49.08 \%$ procyanidins and $1.02 \%$ monomeric flavanols.

\section{Animals and experimental design}

Male Wistar rats (180-200 g) were obtained from the National Laboratory Animal Center, Mahidol University, Salaya, Thailand. All animal experiments were conducted according to the ethical guidelines outlined in the Guide for Care and
Use of Laboratory Animals. Animal facilities and protocols were approved by the Laboratory Animal Care and Use Committee at the Faculty of Veterinary Science, Chulalongkorn University, Thailand. Wistar rats were housed in individual, stainless-steel cages in a room maintained at $25 \pm 1{ }^{\circ} \mathrm{C}$ on a $12 \mathrm{~h}$ light $-12 \mathrm{~h}$ dark cycle. They were fed standard laboratory chow with water ad libitum and fasted overnight before the experiments.

Rats were randomly divided into four groups of six animals each. As shown in Tables 1 and 2 , the composition of the normal diet (ND; American Institute of Nutrition-93 diet) and the HF was prepared following reported procedures ${ }^{(15)}$. For 8 weeks, groups 1 and 2 received the ND and HF, respectively, whereas groups 3 and 4 were fed the HF supplemented with 0.5 and $1.0 \%$ GSE, respectively. Body weight was monitored weekly. At the end of the experiment, the rats were fasted overnight before blood sampling. Blood samples were obtained from the tail vein and collected in tubes with or without heparin-containing solution. These samples were immediately centrifuged $(2500 \mathrm{~g})$, and the plasma was separated and frozen at $-20^{\circ} \mathrm{C}$ until analysed for blood chemistry. The animals were euthanised; the soleus muscle was immediately removed, frozen in liquid $\mathrm{N}_{2}$ and stored at $-70^{\circ} \mathrm{C}$.

\section{Biochemical analysis}

Fructosamine was determined using the nitroblue tetrazolium assay. Plasma adiponectin levels were determined by ELISA. Total serum cholesterol, TAG and HDL-cholesterol levels were assayed by using enzymatic methods. Blood urea $\mathrm{N}$, creatinine, and the activities of alanine and aspartate transaminases (AST) and alkaline phosphatase were determined using an autoanalyser at Service Unit, Faculty of Allied Health Sciences, Chulalongkorn University.

\section{Tissue homogenisation}

The frozen soleus muscles were cut into small pieces $(0 \cdot 2 \mathrm{~g})$ and homogenised in $0.8 \mathrm{ml}$ homogenising buffer containing 250 mm-sucrose, 20 mm-Tris ( $\mathrm{pH} 7.5$ ), 2 mm-EDTA, $0.5 \mathrm{~mm}$ ethylene glycol tetraacetic acid, leupeptin $(20 \mu \mathrm{g} / \mathrm{ml})$, aproti$\operatorname{nin}(10 \mu \mathrm{g} / \mathrm{ml})$, phenylmethylsulfonyl fluoride $(174.2 \mu \mathrm{g} / \mathrm{ml})$, $20 \mathrm{~mm}$-dithiothreitol and 5\% Triton X-100. The homogenate was then centrifuged at $10000 \mathrm{~g}$ for $10 \mathrm{~min}$ at $4^{\circ} \mathrm{C}$. The Bradford assay was used to determine the protein concentration

Table 1. Composition of the experimental diets ( $\mathrm{g} / \mathrm{kg}$ diet)

\begin{tabular}{lcc}
\hline Ingredients & Normal diet & High-fructose diet \\
\hline Casein & 200 & 200 \\
Maize starch & 530 & - \\
Sucrose & 100 & - \\
Fructose & - & 630 \\
Soyabean oil & 70 & 70 \\
Mineral mixture & 35 & 35 \\
Vitamin mixture & 10 & 10 \\
Cellulose powder & 50 & 50 \\
L-Cys & 3 & 3 \\
Choline bitartrate & 2.5 & 2.5 \\
\hline
\end{tabular}


Table 2. Fructosamine, lipid profiles, and kidney and liver function parameters in rats at the end of the experimental periods (8 weeks)

(Mean values with their standard errors, $n 6$ )

\begin{tabular}{|c|c|c|c|c|c|c|c|c|}
\hline \multirow[b]{2}{*}{ Parameters } & \multicolumn{2}{|c|}{ ND } & \multicolumn{2}{|c|}{ HF } & \multicolumn{2}{|c|}{$\mathrm{HF}+0.5 \%$ GSE } & \multicolumn{2}{|c|}{$\mathrm{HF}+1.0 \%$ GSE } \\
\hline & Mean & SEM & Mean & SEM & Mean & SEM & Mean & SEM \\
\hline Fructosamine $(\mathrm{mmol} / \mathrm{l})$ & 0.82 & 0.04 & $0.92^{*}$ & 0.03 & $0.70 \dagger$ & 0.02 & $0.74 \dagger$ & 0.04 \\
\hline Adiponectin $(\mu \mathrm{g} / \mathrm{ml})$ & $16 \cdot 38$ & 1.05 & $9 \cdot 97^{*}$ & 0.79 & $14 \cdot 19 \dagger$ & 0.88 & $13 \cdot 16 \dagger$ & 0.81 \\
\hline Total cholesterol (mg/l) & 860 & 25 & $992^{*}$ & 21 & 994 & 30 & 986 & 27 \\
\hline $\mathrm{TAG}(\mathrm{mg} / \mathrm{l})$ & 448 & 37 & $767^{\star}$ & 92 & $592 \dagger$ & 43 & $572 \dagger$ & 31 \\
\hline HDL-cholesterol (mg/l) & 643 & 28 & 702 & 30 & 686 & 27 & 686 & 19 \\
\hline Blood urea N (mg/l) & 180 & 8.9 & $201 \cdot 7$ & 6.5 & $188 \cdot 3$ & 6 & $196 \cdot 7$ & $6 \cdot 2$ \\
\hline Creatinine (mg/l) & $8 \cdot 2$ & $3 \cdot 1$ & 7.5 & 0.2 & $7 \cdot 0$ & 0.1 & 8.0 & $2 \cdot 6$ \\
\hline ALT (U/l) & 24.50 & $2 \cdot 72$ & $28 \cdot 17$ & 0.95 & $26 \cdot 17$ & $2 \cdot 70$ & $26 \cdot 50$ & 1.43 \\
\hline AST (U/I) & 124.00 & $7 \cdot 68$ & $152 \cdot 17$ & 25.92 & $105.83 \dagger$ & 6.85 & $108.67 \dagger$ & $10 \cdot 99$ \\
\hline ALP (U/I) & $90 \cdot 17$ & 3.03 & 85.00 & 2.03 & 90.50 & 5.90 & 87.00 & 4.18 \\
\hline
\end{tabular}

ND, normal diet; HF, high-fructose diet; GSE, grape seed extract; ALT, alanine transaminase; AST, aspartate transaminase; ALP, alkaline phosphatase.

${ }^{*}$ Mean values were significantly different from those of the ND group $(P<0.05)$

† Mean values were significantly different from those of the HF group $(P<0.05)$.

of the supernatant, which was then stored at $-80^{\circ} \mathrm{C}$ until subjected to Western blot analysis ${ }^{(16)}$.

\section{Western blot analysis}

For detection of anti-IR $\beta$, muscle homogenates containing protein $(5 \mu \mathrm{g} / \mu \mathrm{l})$ were subjected to immunoprecipitation with an anti-IR $\beta$ subunit antibody at $4^{\circ} \mathrm{C}$ overnight, followed by shaking with protein A Sepharose beads according to the manufacturer's instructions. The protein $(35 \mu \mathrm{g})$ was separated by $10 \%$ SDS-PAGE at a constant 70 and $130 \mathrm{~V}$ for the stacking and separating phases, respectively, and transferred by electroblotting onto a PolyScreen polyvinylidene fluoride transfer membrane using a semi-dry transfer cell (Bio-Rad, Hercules, CA, USA) at a constant $100 \mathrm{~V}$ for $2 \mathrm{~h}$ at $4^{\circ} \mathrm{C}$ (Laemmli, 1970). The membrane was then treated sequentially with blocking solution (Tris-buffered saline Tween-20 containing 5\% nonfat, skimmed milk). After three 5 min washes in Tris-buffered saline Tween-20, the membrane was incubated with the primary antibody against IR $\beta$ at $4^{\circ} \mathrm{C}$ overnight, washed and then incubated with the horseradish peroxidase-linked secondary antibody at room temperature for $1 \mathrm{~h}$. After washing, the blotted proteins were visualised using the enhanced chemiluminescence detection system.

For the detection of anti-IRS-1, anti-Akt and anti-GLUT, proteins $(35 \mu \mathrm{g})$ were prepared from muscle homogenates, subjected to SDS-PAGE, transferred to a polyvinylidene fluoride membrane as described earlier and blotted with anti-IRS-1, anti-Akt or anti-GLUT4 antibodies according to the manufacturer's instructions at $4^{\circ} \mathrm{C}$ overnight, washed and then incubated with the horseradish peroxidase-linked secondary antibody at room temperature for $1 \mathrm{~h}$. After washing, the blotted proteins were visualised using enhanced chemiluminescence. Band intensities were quantified by the Bio-Rad ChemiDoc XRS Gel Documentation system ${ }^{(16)}$. The mean value for samples from the ND group on each immunoblot, expressed in densitometry units, was adjusted to a value of 1.0. All experimental sample values are then expressed relative to this adjusted mean value.

\section{Total RNA extraction and RT-PCR}

Total RNA from the soleus muscle was isolated with TRIzol reagent according to the manufacturer's directions. The concentration and purity of the RNA extracted were checked by measuring the absorbance at 260 and $280 \mathrm{~nm}$. Total RNA $(1 \mu \mathrm{g} / \mu \mathrm{l})$ was incubated with $10 \mu \mathrm{m}$-Oligo dT primer at $70^{\circ} \mathrm{C}$ for $5 \mathrm{~min}$ and then reverse transcribed to Complementary DNA in a reaction mixture of $1 \times$ ImProm-II reaction buffer, RNase inhibitor $(1 \mathrm{U} / \mu \mathrm{l}), 0.5 \mathrm{~mm}$-dNTP Mix and ImProm-II RT at $42^{\circ} \mathrm{C}$ for $60 \mathrm{~min}$. The mixture was heated at $70^{\circ} \mathrm{C}$ for $15 \mathrm{~min}$ to terminate the reaction. PCR was performed in a mixture $(20 \mu \mathrm{l})$ containing $2.5 \mu \mathrm{l}$ of $10 \times$ Taq buffer, $5 \mu \mathrm{l}$ reversetranscribed template solution, $1 \mathrm{~mm}-\mathrm{MgCl}_{2}, 10 \mu \mathrm{m}$ each of sense and antisense primers, $0.2 \mathrm{~mm}$-dNTP mix and $1.25 \mathrm{U}$ Taq DNA polymerase.

The specific primer sequences were as follows: hypoxanthine guanine phosphoribosyl transferase (Hprt; GenBank accession no. NM_012583.2), 5'-CAG TCC CAG CGT CGT GAT TA-3' (sense) and $3^{\prime}$-AGC AAG TCT TTC AGT CCT GTC-5' (antisense); adiponectin (GenBank accession no. NM_144744.2), 5'-AAT CCT GCC CAG TCA TGA AG-3' (sense) and $3^{\prime}$-CAT CTC CTG GGT CAC CCT TA-5' (antisense); adiponectin receptor R1 (AdipoR1; GenBank accession no. NM_207587.1), 5'-CTT CTA CTG CTC CCC ACA GC-3' (sense) and $3^{\prime}$-TCC CAG GAA CAC TCC TGC TC-5' (antisense); AMPK- $\alpha 1$ (GenBank accession no. NM_019142.1), 5'-TGT GAC AAG CAC ATT TTC CAA-3' (sense) and $3^{\prime}$-CCG ATC TCT GTG GAG TAG CAG-5' (antisense); glycogen synthase 1 (GS1; GenBank accession no. NM_001109615.1), 5'-TTT TCC TTG GTC CAG TGT CC-3' (sense) and 3'-CGG TGG GCT CAC TTT TGT AT-5' (antisense); glycogen synthase kinase-3 (GSK-3 $\alpha$; GenBank accession no. NM_017344.1), 5'-ACC TAC CCT CAC TAA CTC TTC CTG-3' (sense) and 3'-GTA GAA GGT CCT CAT ACC CCA AAC-5' (antisense). Taq DNA polymerase was activated by a $95^{\circ} \mathrm{C}$ incubation step for $3 \mathrm{~min}$. After an initial denaturation at $95^{\circ} \mathrm{C}$ for $60 \mathrm{~s}$, reactions were performed as follows: for Hprt detection, $60^{\circ} \mathrm{C}$ for $30 \mathrm{~s}$, thirty-five cycles (139 bp); for adiponectin detection, $60^{\circ} \mathrm{C}$ for $30 \mathrm{~s}$, thirty-five cycles ( $215 \mathrm{bp}$ ); for adiponectin receptor 1 
detection, $60^{\circ} \mathrm{C}$ for $30 \mathrm{~s}$, thirty-five cycles (139 bp); for AMPK- $\alpha 1$ detection, $55^{\circ} \mathrm{C}$ for $30 \mathrm{~s}$, thirty-five cycles (134 bp); for GS1 detection, $57^{\circ} \mathrm{C}$ for $30 \mathrm{~s}$, thirty-five cycles (190 bp); for GSK- $3 \alpha, 57^{\circ} \mathrm{C}$ for $30 \mathrm{~s}$, thirty-five cycles (266 bp). The final extension was performed at $72^{\circ} \mathrm{C}$ for $1 \mathrm{~min}$. The products were electrophoresed on a $2 \%$ agarose gel. After the gel was stained with ethidium bromide, the relative density of the bands was measured using the Bio-Rad ChemiDoc XRS Gel Documentation system and Bio-Rad Quantity One 1-D Analysis software. Relative quantification of the PCR products was done after normalisation to the amount of Hprt mRNA.

\section{Glycogen accumulation}

The frozen soleus muscles were homogenised in ice-cold $0.6 \mathrm{M}-\mathrm{HClO}_{4}$. The mixture was immediately centrifuged at $3000 \mathbf{g}$ and analysed for the determination of free glucose in the tissue by the glucose oxidase method. Amyloglucosidase solution $(10 \mathrm{U} / \mathrm{ml})$ in $0.2 \mathrm{M}$-sodium acetate buffer $(\mathrm{pH} 4.8)$ was then mixed and incubated in the mixture at $40^{\circ} \mathrm{C}$ for $2 \mathrm{~h}$. After incubation, the $\mathrm{pH}$ of the mixture was adjusted to 7 and assayed for the determination of total glucose. Free glucose was subtracted from total glucose to obtain glycogen content. Glycogen concentration is expressed as $\mathrm{mg} / \mathrm{g}$ wet tissue $\mathrm{e}^{(17)}$

\section{Statistical analysis}

Data are expressed as means with their standard errors of the mean for animals under each diet. Statistical significance was evaluated using one-way ANOVA followed by Duncan's multiple range test. A $P$ value $<0.05$ was considered statistically significant.

\section{Results}

\section{Biochemical parameters}

Rats fed the HF alone had a markedly elevated plasma fructosamine level. Moreover, the plasma fructosamine level was significantly reduced in the HF groups supplemented with 0.5 and $1.0 \%$ GSE. Furthermore, plasma adiponectin concentration was reduced by $40 \%$ in the HF group, compared with that in the ND group. However, its concentration in rats fed the HF with 0.5 and $1.0 \%$ GSE was increased by approximately 42 and $32 \%$, respectively. There was a significant $(71 \%)$ increase in the serum TAG level of the HF group compared with the ND group, whereas the GSE-fed groups ( 0.5 and $1.0 \%)$ had serum TAG levels that were reduced by 23.0 and $25.0 \%$, respectively, relative to the HF group. Fructose feeding caused a significant increase of $15 \%$ in the total cholesterol level. However, there was no significant difference in total cholesterol levels between the HF group and the HF groups supplemented with GSE. No change in HDL-cholesterol levels, alanine transminase and alkaline phosphatase was observed among the groups at the end of the experiments. Meanwhile, the GSE treatment significantly decreased the AST levels in HF rats supplemented with 0.5 and $1.0 \%$ GSE, compared with the HF group.

\section{Pathway-related proteins in the skeletal muscle}

The expression of proteins in the insulin-signalling pathway for skeletal muscle was measured for IR, IRS-1, Akt and GLUT4. As shown in Fig. 1, HF rats had impaired protein expression for the skeletal muscle insulin signalling pathway as shown by the decrease in the expression of IR by $54 \%$, IRS- 1 by $41 \%$, Akt by $52 \%$ and GLUT 4 by $56 \%$. After 4 weeks of GSE supplementation, there was no increase in the protein expression of IR and IRS-1 among the HF group and the HF ones supplemented with GSE. The Akt protein expression for $\mathrm{HF}$ rats supplemented with 0.5 and $1.0 \%$ GSE increased 1.41- and 1.47-fold when compared with that of the HF group. The HF group supplemented with $0.5 \%$ GSE was slightly increased for GLUT4 protein expression. Moreover, the GLUT4 protein content for the HF group supplemented with $1 \%$ GSE was significantly increased by 1.82 fold when compared with that of the HF group.

\section{Pathway-related mRNA expression in the skeletal muscle}

The mRNA expression levels of adiponectin, AdipoR1 and AMPK- $\alpha 1$ in the soleus muscle are shown in Fig. 2. After being normalised to Hprt1, the relative mRNA level of adiponectin in the HF group was significantly decreased by $50 \%$ when compared with that in the ND group. GSE supplementation $(0.5$ and $1.0 \%)$ significantly ameliorated the changes in the mRNA levels of adiponectin, with the levels close to those of the ND groups. The HF feeding also decreased the skeletal muscle mRNA expression of AdipoR1 by $30 \%$, and supplementation with GSE (0.5 and $1.0 \%)$ enhanced this expression by $26 \%$ compared with the HF group. Moreover, HF feeding also suppressed the mRNA expression of AMPK$\alpha 1$ by $22 \%$ when compared with the ND. For the HF groups supplemented with 0.5 and $1.0 \%$ GSE, this expression was increased by 34 and $39 \%$, respectively, relative to the HF group.

\section{Glycogen synthesis}

As shown in Fig. 3, the mRNA expression of GS1 was lower in the HF group than in the ND group, whereas the supplementation of GSE significantly increased the mRNA levels of GS1 in the HF group. There was no significant difference between the mRNA expression of GSK3 $\alpha$ in the ND group and the HF group. The HF groups supplemented with GSE exhibited significantly lower mRNA expression of GSK3 $\alpha$ when compared with the HF group. In the skeletal muscle, glycogen accumulation in the HF group was lower than that in the ND group by $19 \%$, whereas the HF supplemented with $1.0 \%$ GSE showed a significant increase in glycogen accumulation by $35 \%$. 


\section{Discussion}

We have recently shown that supplementing the diet of HF rats with GSE attenuated hyperglycaemia and hyperinsulinaemia and improved insulin sensitivity ${ }^{(13)}$. In that study, 8 weeks after the diet, the body weight had significantly decreased in rats on an HF with $0.5 \%$ and $1.0 \%$ GSE, relative to that of HF rats. Plasma glucose and plasma insulin significantly increased in these rats; however, $1.0 \%$ GSE resulted in a significant decrease in these levels. Decreased HOMA-IR scores and incremental AUC for glucose were also observed in HF rats supplemented with GSE, compared with untreated HF rats ${ }^{(13)}$. Chronic high-fructose feeding in experimental animals has been reported to produce hypertriacylglycerolaemia by increasing the rate of de novo lipogenesis and TAG synthesis $^{(18)}$. Increased delivery of TAG to the skeletal muscle interferes with glucose utilisation, ultimately leading to hyperglycaemia and hyperinsulinaemia. The results of the present study indicate that GSE might have improved
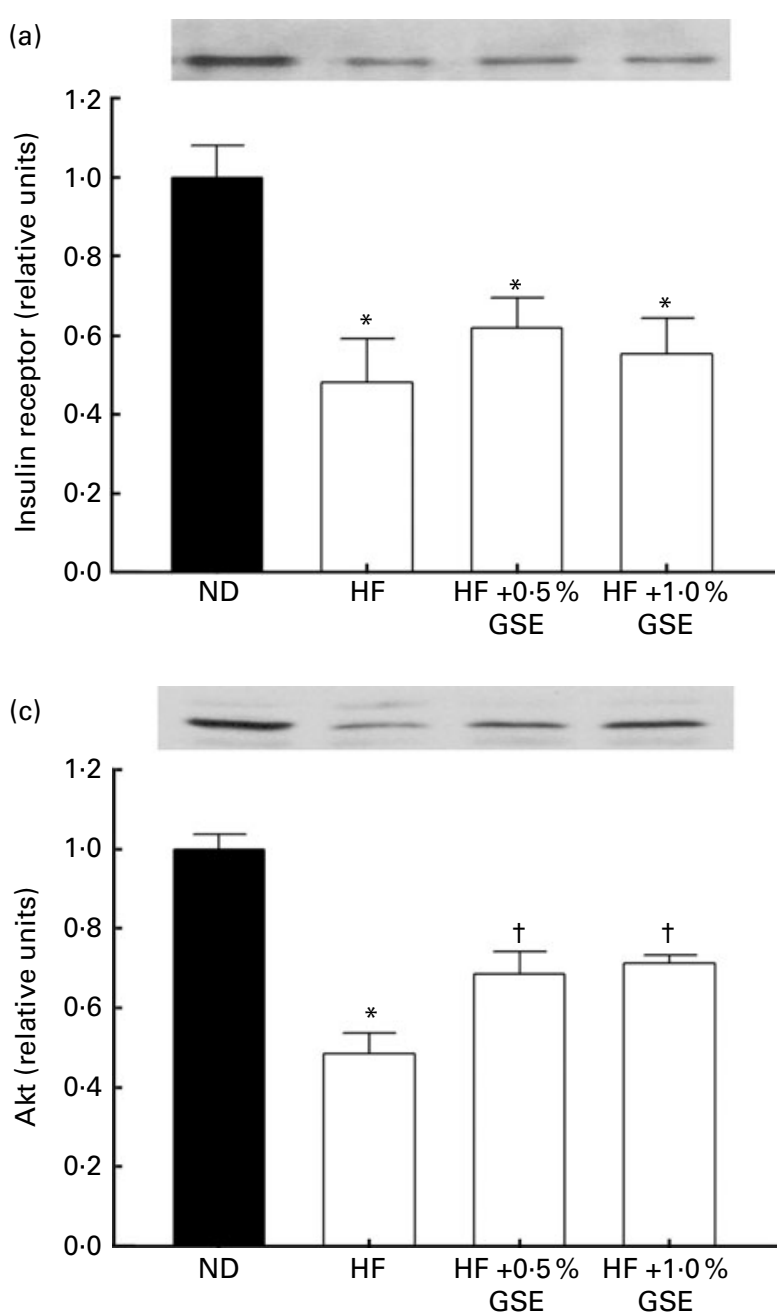

insulin resistance in rats by preventing HF-induced hypertriacylglycerolaemia.

Amadori products such as fructosamine result from the reaction between the carbonyl group of a glucose or fructose and the amino group of a protein to form a Schiff base ${ }^{(19)}$. Thereafter, fructosamine undergoes further protein cross-linking reactions to form advanced glycation end products, which play an important role in diabetic complications through a series of pathological changes such as decreased insulin binding to its receptors ${ }^{(20)}$. Recent findings have revealed that advanced glycation end products directly induce the impairment of insulin action in the muscle by mediating the formation of a multi-molecular complex that consists of receptor for advanced glycation end products (RAGE)/IRS-1 Src and protein kinase C- $\alpha(\operatorname{PKC}-\alpha)^{(21)}$. Generally, fructosamine levels are used to access glycaemic control since they can indicate the accumulation of early (Amadori) glycation products in diabetic patients. The present findings demonstrate that plasma fructosamine was markedly increased in the $\mathrm{HF}$
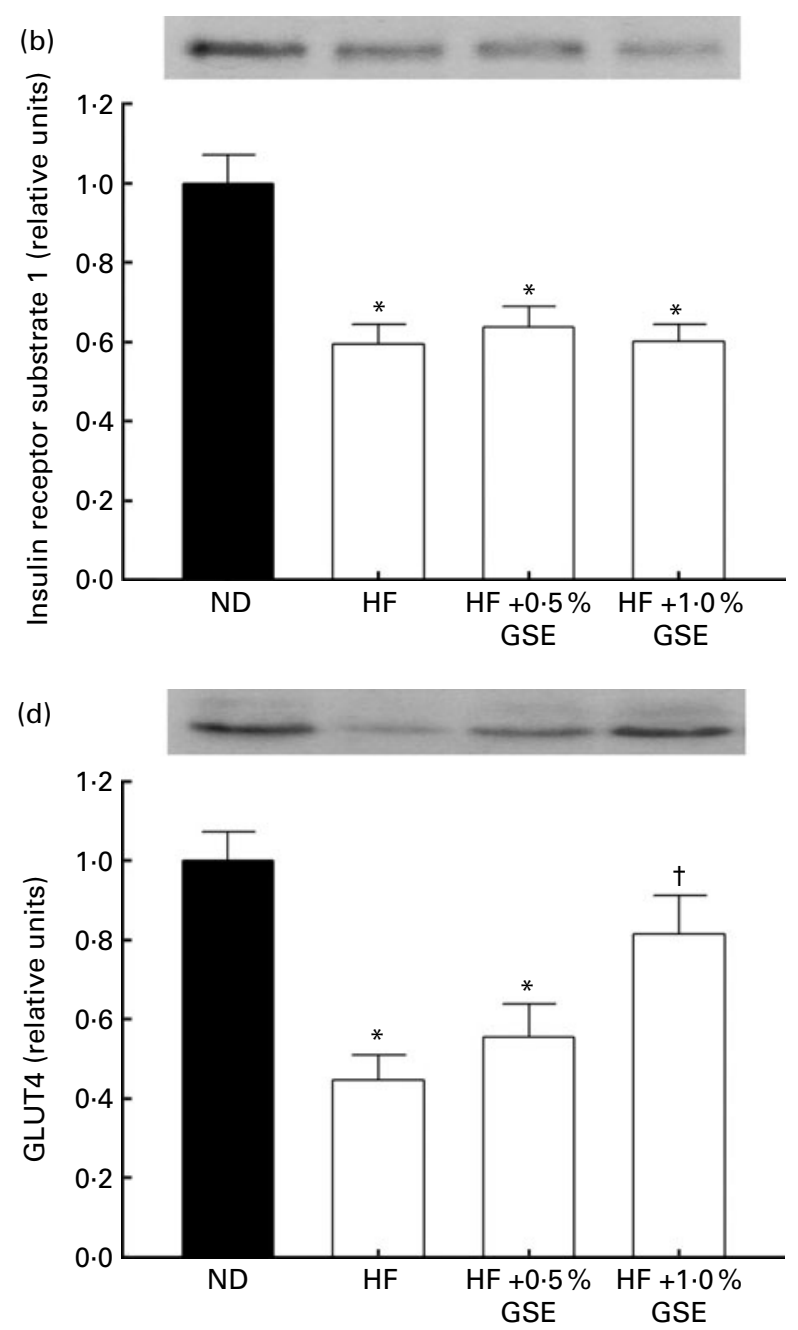

Fig. 1. Effect of grape seed extract (GSE) on the protein expression of (a) insulin receptor, (b) insulin receptor substrate 1, (c) Akt and (d) GLUT4 in the soleus muscle isolated from rats after 8 weeks of the dietary experiment. Values are means, with their standard errors represented by vertical bars $(n 6)$. ${ }^{\star}$ Mean values were significantly different from those of the normal diet (ND) group $(P<0 \cdot 05)$. $†$ Mean values were significantly different from those of the high-fructose diet $(\mathrm{HF})$ group $(P<0.05)$. 
(a)

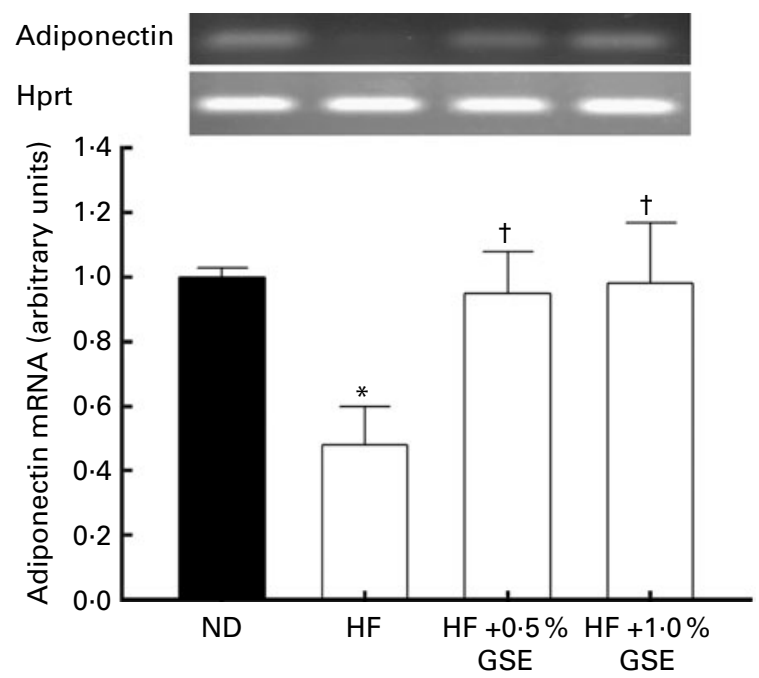

(b)

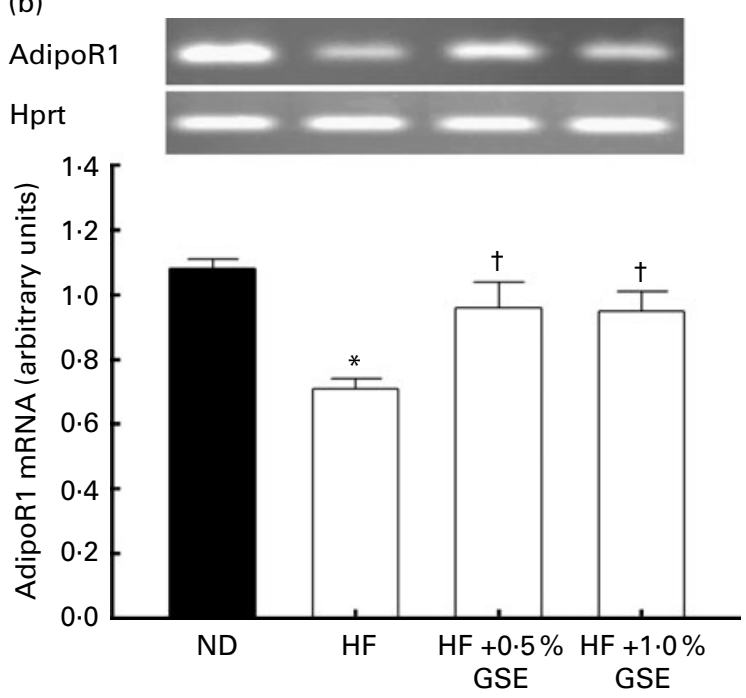

(c) AMPK- $\alpha$

Hprt
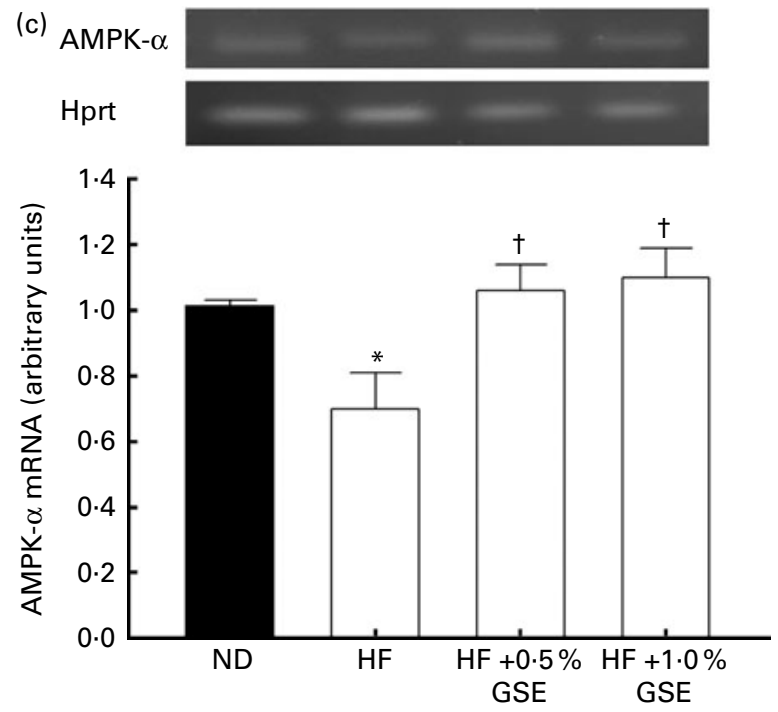

Fig. 2. Effect of grape seed extract (GSE) on the mRNA expression of (a) adiponectin, (b) adiponectin receptor R1 (AdipoR1) and (c) AMP-activated protein kinase- $\alpha$ (AMPK- $\alpha)$ in the soleus muscle isolated from rats after 8 weeks of the experiment. Values are means, with their standard errors represented by vertical bars $(n 6)$. * Mean values were significantly different from those of the normal diet (ND) group $(P<0.05)$. † Mean values were significantly different from those of the high-fructose diet (HF) group $(P<0 \cdot 05)$. Hprt, hypoxanthine guanine phosphoribosyl transferase.

group but decreased in the HF groups supplemented with GSE. Recently, GSE has been shown to inhibit fructosemediated non-enzymatic glycation in vitro ${ }^{(11)}$. The results from the previous study support that the fructosamine-lowering effect may account for the anti-glycation activity of GSE. The inhibitory effect of GSE might decrease advanced glycation end product formation and thereby prevent the progression of diabetic complications.

Creatinine is a product of phosphocreatine degradation and is excreted in the urine. Increased plasma creatinine levels indicate the inability of kidneys to eliminate protein metabolic by-products. In the present study, there was no significant change in creatinine and blood urea $\mathrm{N}$ levels in the HF group, indicating that renal function was unaffected. Changes in liver function parameters reflect hepatocellular damage. In the present study, alanine transaminase and alkaline phosphatase levels were not altered by the HF, indicating that hepatic function in the HF group remained normal. AST levels tended to be higher in the HF group, but it was not significant when compared with those in the ND group. However, GSE significantly lowered AST levels in the supplemented HF groups relative to the untreated HF group. Several studies have described chemical components in GSE that can reverse alanine transaminase and AST alterations in hepatic injury by reducing oxidative stress ${ }^{(22,23)}$. Although the possible AST-lowering mechanisms of GSE in high-fructose-fed rats remain unclear, we suggest that it is possibly related to components having antioxidant activity.

Insulin resistance represents a decrease in the insulin responsiveness of target tissues, namely skeletal muscle, adipose tissue and the liver. Chronic HF feeding directly impairs insulin action on peripheral glucose uptake by reducing the 

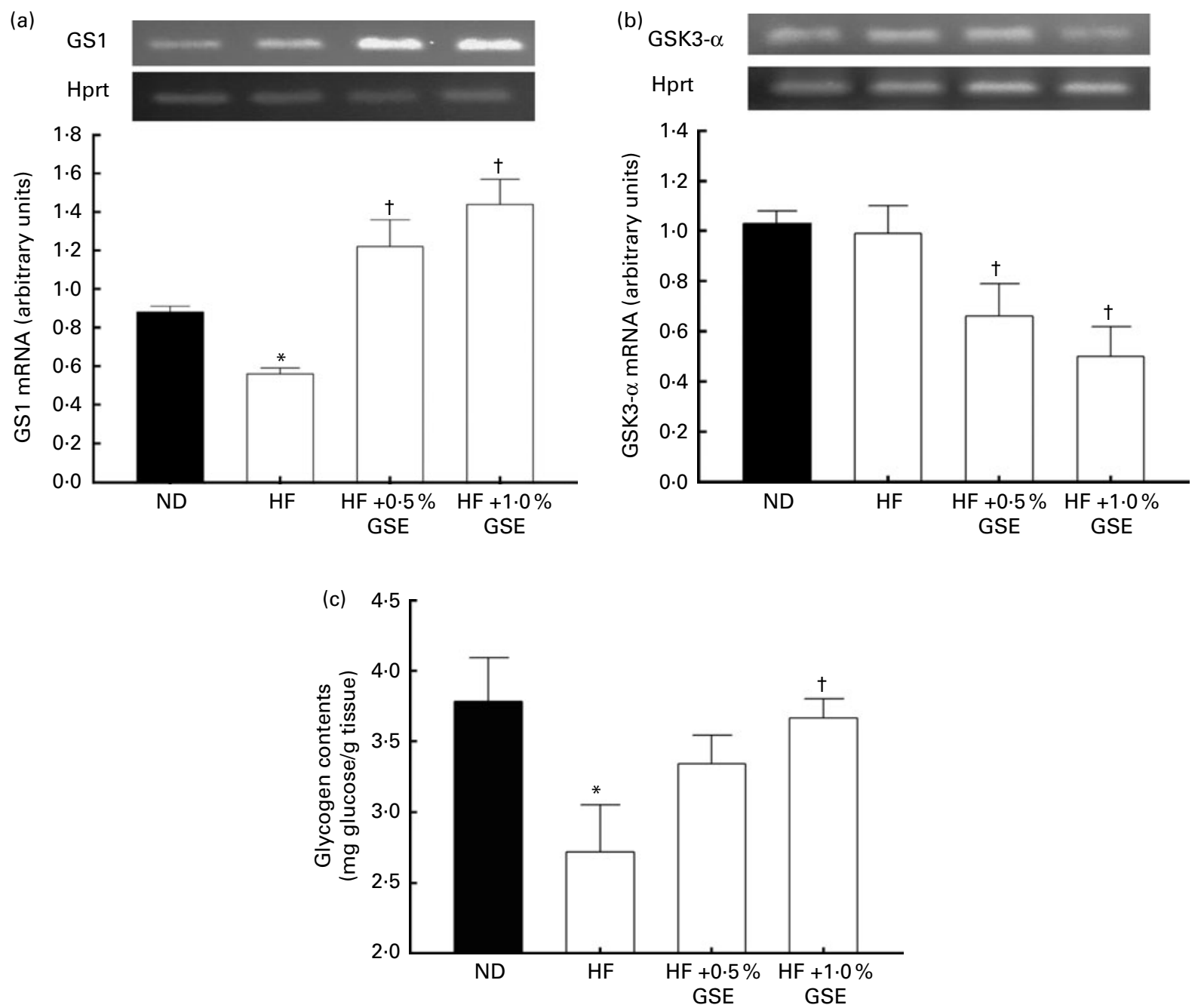

Fig. 3. Effect of grape seed extract (GSE) on the mRNA expression of (a) glycogen synthase-1 (GS1), (b) glycogen synthase kinase-3 (GSK3- $\alpha$ ) and (c) glycogen accumulation in the soleus muscle isolated from rats after 8 weeks of the experiment. Values are means, with their standard errors represented by vertical bars ( $n$ 6). * Mean values were significantly different from those of the normal diet (ND) group $(P<0.05)$. $†$ Mean values were significantly different from those of the high-fructose diet $(\mathrm{HF})$ group $(P<0 \cdot 05)$. Hprt, hypoxanthine guanine phosphoribosyl transferase.

expression of insulin-stimulated proteins such as IR, IRS-1, Akt and GLUT4 ${ }^{(24)}$. We have found that excess fructose interferes with insulin action by altering the levels of these proteins. These results are in good agreement with other studies $^{(24,25)}$. In general, insulin activates its specific receptor, which leads to the activation of IRS-1 and its increased association with phosphoinositide 3-kinase (PI3K), resulting in increased PI3K activity and the activation of protein kinase B or Akt. Finally, the pathway stimulates GLUT4 translocation to the plasma membrane and results in enhanced glucose uptake into the cells ${ }^{(26)}$. The present study showed that GSE improves Akt and GLUT4 expression in the skeletal muscle of $\mathrm{HF}$ rats. This leads to improved insulin sensitivity and hyperglycaemia, providing strong evidence for the potential beneficial effects of GSE in diabetic patients.

Adiponectin, a hormone secreted by adipocytes, plays a protective role against insulin resistance by regulating glucose and lipid metabolism ${ }^{(27)}$. Circulating adiponectin acts primarily in muscle by binding to AdipoR $1^{(28)}$, which activates
AMPK- $\alpha$. AMPK- $\alpha$ plays an important role in the regulation of cellular energy levels and elicits glucose uptake by increasing GLUT4 translocation ${ }^{(29)}$. The reduction in plasma adiponectin levels has been reported to be associated with impaired insulin sensitivity ${ }^{(30)}$. The present results are in accordance with other reports of decreased plasma adiponectin levels after an $\mathrm{HF}^{(31,32)}$, whereas it was markedly corrected by GSE. On the basis of a previous study, we suggest that the improvement in whole-body insulin sensitivity and glycaemic control in HF rats by GSE positively correlates with a rise in plasma adiponectin level ${ }^{(13)}$. Chronic fat and fructose consumption is associated with defects in adiponectin receptor expression in the skeletal muscle, which could contribute to the alterations in glucose metabolism ${ }^{(5)}$. The present results are concordant with reports describing that high consumption of fructose markedly impairs adiponectin receptor expression $^{(5)}$. We found that GSE restores the mRNA expression of AdipoR1 and AMPK- $\alpha$ in HF rats. The increased AdipoR1 expression might enhance adiponectin-binding 
capacity to muscle cells and increase adiponectin effects. Furthermore, the up-regulation of AMPK- $\alpha$ by GSE is likely to promote the effect of insulin-independent stimuli for glucose uptake by activating translocation of GLUT4 to the plasma membrane. Until recently, adipocytes were thought to be the only site for adiponectin synthesis and secretion. However, several studies have now discovered that skeletal muscle cells can synthesise and secrete adiponectin, which then exerts local metabolic effects, at least in part, via elevated glucose uptake ${ }^{(33-35)}$. There is now considerable evidence that a decrease in local adiponectin expression could be at least partly responsible for skeletal muscle insulin resistance $^{(35)}$. The results demonstrated that skeletal muscle adiponectin production is altered in HF rats, and that these changes can be corrected by GSE, suggesting that increased muscle adiponectin expression might partially enhance insulin sensitivity in HF rats.

Skeletal muscle glycogen synthesis is important for blood glucose homeostasis. The ability of insulin to increase glycogen accumulation in muscle involves the stimulation of GLUT and the activation of glycogen synthase ${ }^{(36)}$. The principal step by which insulin stimulates glycogen synthase is via the activation of the IR, which leads to the PI3K-dependent activation of protein kinase B/Akt, inactivation of GSK-3 and increased glycogen synthase (GS) activity ${ }^{(26)}$. A reduction in GSK-3 $\alpha$ expression has been shown to improve insulin action by stimulating glucose uptake and glycogen synthesis ${ }^{(37)}$. In contrast, elevation of GSK3 $\alpha$ generates impaired insulin responsiveness and insulin resistance in the skeletal muscle ${ }^{(37)}$. In the present study, GSE suppressed the mRNA expression of GSK $3 \alpha$ and the elevated mRNA expression of GS1, in agreement with the increased glycogen accumulation and resulting reduction of plasma glucose levels in HF rats.

However, the detailed mechanisms for the induction of impairment in HF rats remain unclear. It is possible that an HF may induce the formation of reactive oxygen species associated with an increase in oxidative stress, which has often been implicated in the pathology of insulin resistance $^{(38)}$. Prolonged oxidative stress can affect the resistance of skeletal muscle insulin signalling, glucose transport and glycogen synthesis ${ }^{(39,40)}$. Increased oxidative stress also inhibits the expression of adiponectin ${ }^{(41)}$. In the present study, GSE was found to comprise procyanidins and monomeric flavanols such as $(+)$-catechin and $(-)$-epicatechin, which have potent antioxidant activities ${ }^{(42-44)}$. Thus, we suggest that these active principles might be acting as antioxidants and helping to prevent the decreased expression of post-receptor-related proteins such as Akt and GLUT4 and of the corresponding mRNA.

Taken together, these results suggest that GSE ameliorates defective insulin and adiponectin signalling pathways in the skeletal muscle of HF rats. Moreover, the present study provided evidence for the molecular mechanisms by which GSE prevents high-fructose-induced insulin resistance.

\section{Acknowledgements}

The present study was financially supported by the 90th Anniversary of Chulalongkorn University Fund (Ratchadaphiseksomphot Endowment Fund). The authors would like to thank the Medical Food Research and Development Center, which has been financially and institutionally supported by the Chulalongkorn University. The authors' contributions were as follows: A. M., S. Y.-a., S. A. developed the concept and designed the study. In particular, A. M., W. So., W. Su. performed the study, collected and analysed the data; S. A. wrote the manuscript. The authors declare that they have no conflicts of interest.

\section{References}

1. Gerrits PM \& Tsalikian E (1993) Diabetes and fructose metabolism. Am J Clin Nutr 58, 796S-799S.

2. Chidambaram J \& Carani Venkatraman A (2007) Myricetin, a naturally occurring flavonol, ameliorates insulin resistance induced by a high-fructose diet in rats. Life Sci $\mathbf{8 1}$, 1479-1488.

3. Qin B, Polansky MM, Harry D, et al. (2010) Green tea polyphenols improve cardiac muscle mRNA and protein levels of signal pathways related to insulin and lipid metabolism and inflammation in insulin-resistant rats. Mol Nutr Food Res 54, Suppl. 1, S14-S23.

4. Havel PJ (2002) Control of energy homeostasis and insulin action by adipocyte hormones: leptin, acylation stimulating protein, and adiponectin. Curr Opin Lipidol 13, 51-59.

5. Bonnard C, Durand A, Vidal H, et al. (2008) Changes in adiponectin, its receptors and AMPK activity in tissues of diet-induced diabetic mice. Diabetes Metab 34, 52-61.

6. Kadowaki T \& Yamauchi $\mathrm{T}$ (2005) Adiponectin and adiponectin receptors. Endocr Rev 26, 439-451.

7. Moreno DA, Ilic N, Poulev A, et al. (2003) Inhibitory effects of grape seed extract on lipases. Nutrition 19, 876-879.

8. Terra X, Montagut G, Bustos M, et al. (2009) Grape-seed procyanidins prevent low-grade inflammation by modulating cytokine expression in rats fed a high-fat diet. $J$ Nutr Biochem 20, 210-218.

9. Mayer R, Stecher G, Wuerzner R, et al. (2008) Proanthocyanidins: target compounds as antibacterial agents. J Agric Food Chem 56, 6959-6966.

10. Pinent M, Blay M, Bladé MC, et al. (2004) Grape seedderived procyanidins have an antihyperglycemic effect in streptozotocin-induced diabetic rats and insulinomimetic activity in insulin-sensitive cell lines. Endocrinology $\mathbf{1 4 5}$, 4985-4990.

11. Adisakwattana S, Jiphimai P, Prutanopajai P, et al. (2010) Evaluation of $\alpha$-glucosidase, $\alpha$-amylase and protein glycation inhibitory activities of edible plants. Int J Food Sci Nutr 61, 295-305.

12. Montagut G, Onnockx S, Vaqué M, et al. (2010) Oligomers of grape-seed procyanidin extract activate the insulin receptor and key targets of the pathway differently from insulin. J Nutr Biochem 21, 476-481.

13. Suwannaphet W, Meeprom A, Yibchok-Anun S, et al. (2010) Preventive effect of grape seed extract against high-fructose diet-induced insulin resistance and oxidative stress in rats. Food Chem Toxicol 48, 1853-1185.

14. Saito M, Hosoyama H, Ariga T, et al. (1998) Antiulcer activity of grape seed extract and procyanidins. J Agric Food Chem 46, $1460-1464$. 
15. Guo H, Ling W, Wang Q, et al. (2007) Effect of anthocyaninrich extract from black rice (Oryza sativa L. indica) on hyperlipidemia and insulin resistance in fructose-fed rats. Plant Foods Hum Nutr 62, 1-6.

16. Liu IM, Tzeng TF, Liou SS, et al. (2007) Myricetin, a naturally occurring flavonol, ameliorates insulin resistance induced by a high-fructose diet in rats. Life Sci 81, 1479-1488.

17. Adisakwattana S, Roengsamran S, Hsu WH, et al. (2005) Mechanisms of antihyperglycemic effect of p-methoxycinnamic acid in normal and streptozotocin-induced diabetic rats. Life Sci $\mathbf{7 8}, 406-412$.

18. Basciano H, Federico L \& Adeli K (2005) Fructose, insulin resistance, and metabolic dyslipidemia. Nutr Metab (Lond) 2, 5.

19. Ahmed N (2005) Advanced glycation endproducts - role in pathology of diabetic complications. Diabetes Res Clin Pract 67, 3-21.

20. Takagi Y, Kashiwagi A, Tanaka Y, et al. (1995) Significance of fructose-induced protein oxidation and formation of advanced glycation end product. $J$ Diabetes Complications 9, 87-91.

21. Cassese A, Esposito I, Fiory F, et al. (2008) In skeletal muscle advanced glycation end products (AGEs) inhibit insulin action and induce the formation of multimolecular complexes including the receptor for AGEs. J Biol Chem 283, 36088-36099.

22. Dulundu E, Ozel Y, Topaloglu U, et al. (2007) Grape seed extract reduces oxidative stress and fibrosis in experimental biliary obstruction. J Gastroenterol Hepatol 22, 885-892.

23. Khoshbaten M, Aliasgarzadeh A, Masnadi K, et al. (2010) Grape seed extract to improve liver function in patients with nonalcoholic fatty liver change. Saudi J Gastroenterol 16, 194-197.

24. Tsai HY, Wu LY \& Hwang LS (2008) Effect of a proanthocyanidin-rich extract from longan flower on markers of metabolic syndrome in fructose-fed rats. J Agric Food Chem 56, 11018-11024.

25. Cao H, Hininger-Favier I, Kelly MA, et al. (2007) Green tea polyphenol extract regulates the expression of genes involved in glucose uptake and in rats fed a high fructose diet. J Agric Food Chem 55, 6372-6378.

26. Musi N \& Goodyear LJ (2006) Insulin resistance and improvements in signal transduction. Endocrine 29, 73-80.

27. Lindsay RS, Funahashi T, Hanson RL, et al. (2002) Adiponectin and development of type 2 diabetes in the Pima Indian population. Lancet 360, 57-58.

28. Yamauchi T, Kamon J, Ito Y, et al. (2003) Cloning of adiponectin receptors that mediate antidiabetic metabolic effects. Nature 423, 762-769.

29. Long YC \& Zierath JR (2006) AMP-activated protein kinase signaling in metabolic regulation. J Clin Invest $\mathbf{1 6}$, $1776-1783$.

30. Stefan N, Vozarova B, Funahashi T, et al. (2002) Plasma adiponectin concentration is associated with skeletal muscle insulin receptor tyrosine phosphorylation, and low plasma concentration precedes a decrease in whole-body insulin sensitivity in humans. Diabetes 51, 1884-1888.

31. Shih CC, Lin CH, Lin WL, et al. (2009) Momordica charantia extract on insulin resistance and the skeletal muscle GLUT4 protein in fructose-fed rats. J Ethnopharmacol 123, 82-90.

32. Qin B, Polansky MM \& Anderson RA (2010) Cinnamon extract regulates plasma levels of adipose-derived factors and expression of multiple genes related to carbohydrate metabolism and lipogenesis in adipose tissue of fructosefed rats. Horm Metab Res 42, 187-193.

33. Delaigle AM, Senou M, Guiot Y, et al. (2006) Induction of adiponectin in skeletal muscle of type 2 diabetic mice: in vivo and in vitro studies. Diabetologia 49, 1311-1323.

34. Yang B, Chen L, Qian Y, et al. (2006) Changes of skeletal muscle adiponectin content in diet-induced insulin resistant rats. Biochem Biophys Res Commun 341, 209-217.

35. Liu Y, Chewchuk S, Lavigne C, et al. (2009) Functional significance of skeletal muscle adiponectin production, changes in animal models of obesity and diabetes, and regulation by rosiglitazone treatment. Am J Physiol Endocrinol Metab 297, E657-E664.

36. Lai YC, Stuenaes JT, Kuo CH, et al. (2010) Insulin-stimulated glycogen synthesis and glycogen synthase activation after electrical stimulation of epitrochlearis muscles with different initial glycogen contents. Arch Physiol Biochem 116, $116-127$

37. Ciaraldi TP, Nikoulina SE, Bandukwala RA, et al. (2007) Role of glycogen synthase kinase-3 alpha in insulin action in cultured human skeletal muscle cells. Endocrinology 148, 4393-4399.

38. Faure P, Rossini E, Lafond JL, et al. (1997) Vitamin E improves the free radical defense system potential and insulin sensitivity of rats fed high fructose diets. J Nutr 127, 103-107.

39. Dokken BB, Saengsirisuwan V, Kim JS, et al. (2008) Oxidative stress-induced insulin resistance in rat skeletal muscle: role of glycogen synthase kinase-3. Am J Physiol Endocrinol Metab 294, E615-E621.

40. Archuleta TL, Lemieux AM, Saengsirisuwan V, et al. (2009) Oxidant stress-induced loss of IRS- 1 and IRS- 2 proteins in rat skeletal muscle: role of p38 MAPK. Free Radic Biol Med 47, 1486-1493.

41. Furukawa S, Fujita T, Shimabukuro M, et al. (2004) Increased oxidative stress in obesity and its impact on metabolic syndrome. J Clin Invest 114, 1752-1761.

42. Yilmaz Y \& Toledo RT (2004) Major flavonoids in grape seeds and skins: antioxidant capacity of catechin, epicatechin, and gallic acid. J Agric Food Chem 52, 255-260.

43. Simonetti P, Ciappellano S, Gardana C, et al. (2002) Procyanidins from Vitis vinifera seeds: in vivo effects on oxidative stress. J Agric Food Chem 50, 6217-6221.

44. Shi J, Yu J, Pohorly JE, et al. (2003) Polyphenolics in grape seeds - biochemistry and functionality. J Med Food 6 , 291-299. 